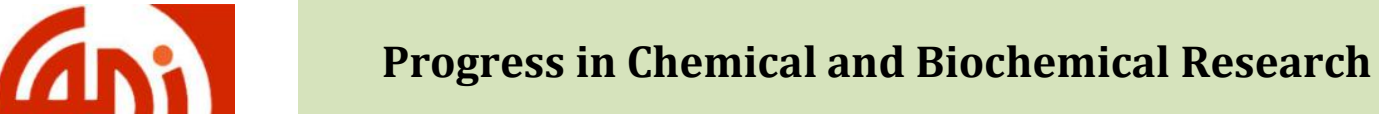

Journal homepage: www.pcbiochemres.com

Original Research Article

\section{Antimicrobials and Enterobacterial Repetitive Intergenic Consensus (ERIC) Polymerase Chain Reaction (PCR) patterns of nosocomial Serratia marcescens isolates: A One Year Prospective Study (June 2013-May 2014) in a Rural Hospital in the Republic of Trinidad and Tobago}

\section{Camille Elliott*, Angel Justiz Vaillant}

Department of Para-Clinical Sciences, Pathology and Microbiology Unit, Faculty of Medical Sciences, University of the West Indies, Eric Williams Medical Complex, Mt. Hope Hospital, Champs Fleurs, Trinidad and Tobago, West Indies.

\begin{tabular}{l} 
A R T I C L E I N F O \\
Article history: \\
Submitted: 2020-02-07 \\
Revised: 2020-03-05 \\
Accepted: 2020-03-11 \\
Published: 2020-04-23 \\
Manuscript ID: PCBR-2002-1081 \\
\hline K E Y W O R D S \\
Antibiotic resistance, \\
Antimicrobials, \\
ERIC PCR-based Fingerprinting \\
method, \\
Nosocomial infection \\
Serratia marcescens
\end{tabular}

A B S T R A C T

Background: Serratia marcescens is a gram-negative bacterium from family of Enterobacteriaceae. It is a human pathogen that is involved in nosocomial infection outbreaks that have proven difficult to manage. PCR-based techniques are suitable given the genus. Serratia has a higher GC content than many other members of the Enterobacteriaceae. The ERIC PCR-based fingerprinting method was used to study the PCR patterns of clinical Serratia marcescens isolates and antimicrobial susceptibility profiles.

Methods: Surveillance was conducted for nosocomial cases of interest and the nosocomial pathogens were retrieved for identification via morphological and additional biochemical tests. Additionally, antimicrobial susceptibility tests and ERIC Polymerase Chain Reaction (PCR) based fingerprinting molecular method was done on the clinical $S$. marcescens isolates.

Results: From the study, five different strains of the clinical Serratia marcescens isolates were recovered and similarly five distinct susceptibility patterns were observed from the clinical $S$. marcescens isolates indicating consistency in the number of strains present in the clinical S. marcescens isolates.

Conclusions: ERIC PCR fingerprinting base technique; a simple, rapid and cheap method for the determination of genetic relatedness between Serratia marcescens isolates can be applied for the thorough evaluation of nosocomial outbreaks to detect the source of infection and control the spreading of the infection.

* Corresponding author: Camille Elliott

Tel number: +1(868) 377-3804, E-mail: camille_basketball@yahoo.com

DOI: $10.33945 /$ SAMI/PCBR.2020.2.4

(C) 2020 by SPC (Sami Publishing Company) 


\section{INTRODUCTION}

Serratia marcescens, is a gram-negative bacillus facultative anaerobic rod belonging to the family Enterobacteriaceae. The bacterium may colonize the nasopharynx and the gut and occasionally causes invasive infections such as bloodstream infection, pneumonia and meningitis; and non-invasive infections including urinary sepsis and conjunctivitis in neonates [1]. It is a well-recognized nosocomial pathogen, a frequent agent of catheterizationassociated bacteriuria [2]. There have been often reports of $S$. marcescens outbreaks in intensive care and neonatal care units[3-6]. It is being transmitted predominantly through patient to patient contact [6].

Outbreaks of infection caused by $S$. marcescens have been reported quite frequently [3,7-9]. Hence, it is imperative that clinicians differentiate between individual isolates to identify potential sources of contamination. S. marcescens was considered originally to be an innocuous, non-pathogen saprophytic water organism. Genotyping of this organism has been carried out using a variety of methodologies $[10,11]$ but many of these approaches are impractical in clinical settings due to various technical demands and time constraints. A DNA fingerprinting strategy based on the PCR amplification of variable-length chromosomal sequences with a variety of primers was reported. One of these approaches, known as random-amplified polymorphic DNA assay, is based on the use of simple arbitrary primers in a PCR of low stringency to amplify segments of the genome and has been used successfully for the typing of several bacterial species[12]. As evidenced by the numerous reports in the literature, the popularity of PCR-based typing methods is rapidly increasing as the method of choice due to the speed at which results are obtained [13].

Traditional methods used for the typing of $S$. marcescens are often based on phenotypic characteristics. PCR have been used for typing $S$. marcescens and it was concluded that PCR finger printing base technique; a simple, rapid and cheap method for the determination of genetic relatedness between Serratia marcescens isolates, and it can be applied for the thorough evaluation of nosocomial outbreaks to detect the source of infection and control the spreading of the outbreak. It has shown a high degree of discriminatory potential and reproducibility $[3,5,13]$. The aim of this study was to use the ERIC based PCR fingerprinting technique to study clinical isolates of $S$. marcescens from hospitalized patients and to investigate antimicrobial susceptibility profiles.

\section{METHODS}

Clinical S. marcescens isolates were retrieved from surveillance over a one-year study period from June 2013 to May 2014 at a Regional Hospital in South West district of Trinidad and Tobago. The nosocomial pathogens of $S$. marcescens were isolated from nosocomial bloodstream infections that had occurred on the neonatal wards during the outbreak. Their susceptibilities to Amikacin (AK), Aztreonam (ATM), Ceftazidime (CAZ), Cefepime (FEP), Ciprofloxacin (CIP), Gentamycin (GM), Piperacillin/Tazobactam (TZP), Tetracycline (TE), Tobramycin (TOB), Amoxicillin/Clavulanic acid (AMC), Amoxillin (AML), Cefaclor (CEC) and Tigecycline (TGC) were determined by Antimicrobial disk diffusion method, according to the procedures suggested by the National Committee for Clinical Laboratory Standards.

\section{DNA extraction technique}

Total DNA isolation and agarose gel electrophoresis were carried out under standard conditions. Documentation of electrophoresed gels was performed using an ultra violet trans-illumination photo documentation system according to the manufacturer's guidelines. Reagents and primers were purchased from Integrated DNA Technologies, Coralville, IA, USA.

\section{ERIC PCR-based fingerprinting technique}

Amplification of DNA was performed in a PHC-3 thermal cycler, with temperature ramping as follows: $95^{\circ} \mathrm{C}$ for 5 minutes to denature template; four lowstringency cycles of $94^{\circ} \mathrm{C}$ for 1 minute, $26^{\circ} \mathrm{C}$ for 1 minute, and $72^{\circ} \mathrm{C}$ for 2 minutes; 40 cycles of $94^{\circ} \mathrm{C}$ for $30 \mathrm{~s}, 40^{\circ} \mathrm{C}$ for $30 \mathrm{~s}$, and $72^{\circ} \mathrm{C}$ for 1 minute and finally, $72^{\circ} \mathrm{C}$ for 10 minutes $-3^{\prime}$ primers, according to the protocol originally described [13]. The reaction mixture (100-,lA volumes) contained $1 \mathrm{U}$ of Taq polymerase, by polymerase chain reaction, using the ERIC1 (5'-TGAATCCCCAGGAGCTTACAT-3') and ERIC2 (5'- AAGTAAGTGACTGGGGTGAGCG-3') $10 \mathrm{mM}$ Tris (pH 8.3), $50 \mathrm{mM} \mathrm{KCl}, 2.5 \mathrm{mM} \mathrm{MgCl}, 0.01 \%$ (wt/vol) gelatin, 250 puM (each) deoxynucleoside triphosphates, and 1 puM single primer. Negative 
controls were prepared, with no addition of template DNA. Amplification products $(10 \mathrm{pA})$ were separated by agarose gel electrophoresis in $1.6 \%$ agarose gels in Tris-borate-EDTA buffer containing ethidium bromide $(1 \mathrm{pLg} / \mathrm{ml})$, at $30 \mathrm{~V}$ for $6 \mathrm{~h}$, and visualized by ultra violet trans-illumination and image was saved in a pen drive for further analysis.

All samples were prepared and examined on at least three separate occasions. The PCR patterns were identical based on similar numbers and matching positions of all major bands. Small differences in the intensities of faint bands were ignored. Reaction conditions and primers used were based on previous studies[13-15].

\section{Identification of bacterial isolates}

Additional biochemical tests were performed based on the morphology of the isolated bacteria to delineate the bacteria's identity. The following biochemical tests were used to confirm the identity of S. marcescens; motility test and facultative anaerobe test. Also S. marcescens were observed to produce red pigment (prodigiosin) and tested positive for glucose, sucrose, maltose and mannitol. This microorganism isolates tested negative in both the hydrogen sulphide test and methyl red test. However, the S. marcescens isolates puM single primer tested positive for Voges- Proskauer, citrate and catalase tests but it tested negative for oxidase and indole tests. S. marcescens isolates were observed to be positive for nitrate and lipase tests.

\section{Data analysis}

Descriptive statistics was employed to show the incidence of $S$. marcescens that was calculated by dividing the number of $S$. marcescens isolates from diseased patients by the total number of forty-five nosocomial blood isolates, and then the product was multiply by 100 . Data collected from all nosocomial infections were entered in a Microsoft ${ }^{\circledR}$ Access 2010 data bank. Data on all infections and microbial isolates, for statistical analysis, IBM SPSS ${ }^{\circ}$ Statistics (version 20) was used.

\section{Ethics}

Ethical approvals were granted from the members of the Ethic Committee of the Faculty of Medical Science and South West Regional Health Authority Ethic governing the San Fernando Regional Hospital in Trinidad and Tobago.

\section{RESULTS}

Table 1 shows the susceptibility patterns of the outbreak at NICU. All strains of Serratia marcescens were multi-resistant and showed different antimicrobial susceptibility patterns. One hundred (100) percent of the isolates were resistant to AML, AMC and CEC as determined by Antimicrobial disk diffusion method, according to the procedures suggested by the National Committee for Clinical Laboratory Standards. Over $90 \%$ of the isolates were sensitive to ATM, CAZ, CIP, TZP and TE.

Potential virulence factors involved in the pathogenicity of $S$. marcescens are; a nuclease, proteases, the haemolysin and lecitinase; all of which are made and secreted by this microorganism[2]. A $56-\mathrm{kDa}$ serine protease from $S$. marcescens promote keratitis by cleaving IgG, IgA, and lysozyme[16]. The S. marcescens haemolysin proteins ShlB and ShlA, exhibit protein sequence homologues in Haemophilus ducreyi, Proteus mirabilis, Yersinia enterocolytica, Yersinia pestis, Edwardsiella tarda, Xylella fastidiosa and Photorhabdus luminescens. The family of Serratia type pore forming toxins has a unique secretory mechanism that has been described as type Vsecretion system. The haemolysin causes haemolysis of human and animal erythrocytes [17] and the release of the inflammatory mediators from leukocytes, such as histamine and leukotrienes, which participate in the inflammatory and immune response by increasing the vascular permeability, edema, and granulocyte accumulation contributing to the virulence of Serratia marcescens[18]. 
Table 1. Antibiogram characteristics of outbreak- related isolates of Serratia marcescens in a neonatal intensive care unit (NICU) at Trinidad and Tobago, 2013 - 2014

\begin{tabular}{ccccccccc}
\hline Strains & Age & AK & FEP & GM & TOB & CN & TGC & Patterns \\
\hline 1 & 28 weeks & $\mathrm{R}$ & $\mathrm{S}$ & $\mathrm{R}$ & $\mathrm{R}$ & $\mathrm{S}$ & $\mathrm{S}$ & $\mathrm{III}$ \\
2 & 44 weeks & $\mathrm{S}$ & $\mathrm{S}$ & $\mathrm{S}$ & $\mathrm{S}$ & $\mathrm{R}$ & $\mathrm{R}$ & $\mathrm{I}$ \\
3 & 35 weeks & $\mathrm{S}$ & $\mathrm{S}$ & $\mathrm{S}$ & $\mathrm{S}$ & $\mathrm{R}$ & $\mathrm{R}$ & $\mathrm{I}$ \\
4 & 28 weeks & $\mathrm{S}$ & $\mathrm{S}$ & $\mathrm{S}$ & $\mathrm{S}$ & $\mathrm{R}$ & $\mathrm{R}$ & $\mathrm{I}$ \\
5 & 41 weeks & $\mathrm{S}$ & $\mathrm{R}$ & $\mathrm{R}$ & $\mathrm{R}$ & $\mathrm{R}$ & $\mathrm{R}$ & $\mathrm{IV}$ \\
6 & 3 weeks & $\mathrm{R}$ & $\mathrm{S}$ & $\mathrm{R}$ & $\mathrm{R}$ & $\mathrm{S}$ & $\mathrm{R}$ & $\mathrm{II}$ \\
7 & 8 days & $\mathrm{R}$ & $\mathrm{S}$ & $\mathrm{R}$ & $\mathrm{R}$ & $\mathrm{S}$ & $\mathrm{R}$ & $\mathrm{II}$ \\
8 & 35 weeks & $\mathrm{R}$ & $\mathrm{R}$ & $\mathrm{S}$ & $\mathrm{R}$ & $\mathrm{R}$ & $\mathrm{S}$ & $\mathrm{V}$ \\
9 & 8 days & $\mathrm{R}$ & $\mathrm{R}$ & $\mathrm{S}$ & $\mathrm{R}$ & $\mathrm{R}$ & $\mathrm{S}$ & $\mathrm{V}$ \\
10 & 28 weeks & $\mathrm{R}$ & $\mathrm{S}$ & $\mathrm{R}$ & $\mathrm{R}$ & $\mathrm{S}$ & $\mathrm{S}$ & $\mathrm{III}$ \\
\hline
\end{tabular}

\section{Serratia marcescens Isolates}

From Fig. 1 above: the two ends represent the 1 kilo base ladders. The lanes numbered one (1) to ten (10) represent the Serratia marcescens organisms in chronological order. Lanes 2, 3 and 4 represents band pattern 1. Lanes 6 and 7 represents band pattern 2. Lane 5 represents pattern 4. Lanes 1 and 10 represent band pattern 3. Lane 8 and 9 represents band pattern 5 .

From the PCR typing technique and antimicrobial susceptibility profile testing, nosocomial patients were placed on treatment plans and were equally monitored to determine the outcome of their treatments, including the infection control measures instituted during their management.

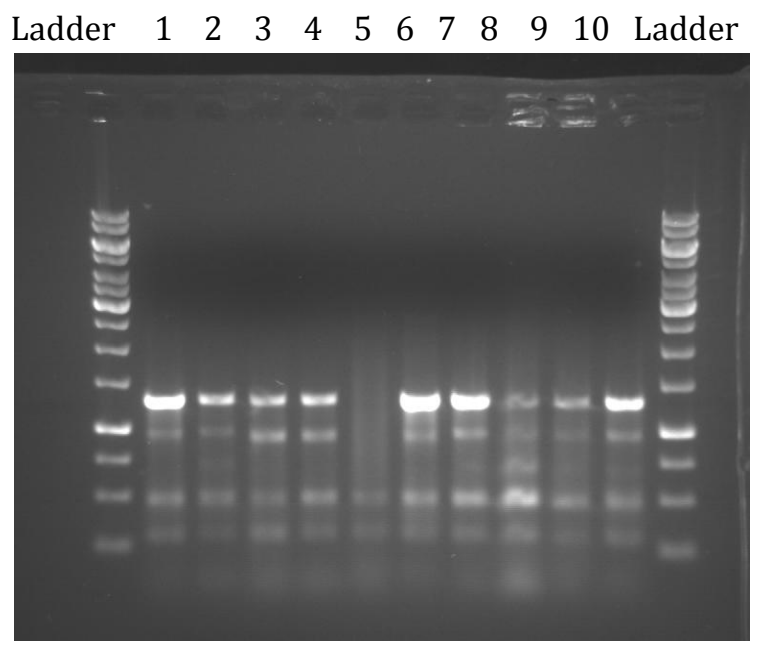

Fig. 1. Showing PCR Band Patterns of

\section{DISCUSSION}

Forty-five nosocomial blood isolates were retrieved with Serratia marcescens (10) nosocomial pathogens being the most frequent and representing $22.2 \%$ $(10 / 45)$. Other retrieved nosocomial pathogens included: Klebsiella spp, methicillin resistant coagulase negative Staphylococcus, Escherichia coli, Streptococcus spp, Enterobacter spp, Acinetobacter spp, Pseudomonas aeruginosa, Staphylococcus spp, Epidermidis aerogenes and Enterococcus spp. Serratia marcescens was commonly found in the bloodstream of hospitalized neonatals admitted at NICU. The ERIC1 and ERIC2 primers successfully typed all isolates examined via PCR- based molecular typing method. Five different patterns were observed for all clinical isolates.

The two ends were 1 kilo base ladder and within the ladders were ten Serratia marcescens isolates encoded 1 - 10; that were retrieved from the neonatal wards. The occurrence of this outbreak of Serratia marcescens was the result of the spread of five epidemic strains on the neonatal wards. No potential environmental sources of infections were identified, although it has been previously reported [5]. However, S. marcescens survives well in moist hospital environments. It was reported the use of pulsed-field gel electrophoresis, random amplification of polymorphic DNA-polymerase chain reaction and plasmid DNA typing to investigate an outbreak of infection in a NICU[19].

In the literature appears that the pulsed-field gel electrophoresis typing was used to analyze an 
outbreak of $S$. marcescens in a NICU. The authors concluded that cross-transmission via transient contamination of hands was the major route of transmission for the outbreak [20]. We believe that the $S$. marcescens outbreak in the NICU at a rural hospital in Trinidad and Tobago was due to a crosscontamination involving the hands of health care professional caring for very sick neonates. Several research groups reported that samples from environment and hands of health care workers were collected and cultured to reveal or identify the source of contamination with Serratia marcescens causing outbreaks. It was found breaks in aseptic technique, poor and decreased frequency of hand-washing among doctors caring for the patients. In addition, hand washing with a Serratia marcescenscontaminated soap pump made the hands of health care workers more likely to be contaminated with the bacterium[20-23]. Genotyping of the $S$. marcescens isolates by pulse-field gel electrophoresis or PCR-based methods should be performed as a gold standard [24].

It was reported that the polymerase chain reaction (PCR)-based procedures of randomly amplified polymorphic DNA (RAPD) and repetitive element (RE)-based PCR were used to amplify total DNA from 62 clinical Serratia marcescens isolates. Using the ERIC, REP and PGRS primers, 19, 54 and 60 unique genotypic profiles were yielded, respectively[6]. These techniques are useful for discriminating strain differences among isolates of $S$. marcescens and the quantity of differentiation depends on the primer used. PCR-based procedures should prove useful for routine surveillance and for examining outbreaks of this bacterium in clinical settings[13].

In the susceptibility test results Amoxicillin (AML), Amoxicillin/Clavulanic acid (AMC), and Cefaclor (CEC), were found to be mostly resistant antibiotics to deal with $S$. marcescens. Bollmann R et al, reported that multi-drug resistance, even to Cephalosporin's of

\section{REFERENCES}

[1] V. Adamson, P. Mitt, H. Pisarev, T. Metsvaht, K. Telling, P. Naaber, M. Maimets, Prolonged outbreak of Serratia marcescens in Tartu University Hospital: a case-control study, BMC Infect. Dis. 12 (2012) 281.

[2] R. Hertle, H. Schwarz, Serratia marcescens internalization and replication in human bladder epithelial cells, BMC Infect. Dis. 4 (2004) 16. the third generation and Amikacin, was characteristically seen for all $S$. marcescens strains[25].

Nosocomial outbreaks of Serratia marcescens infections have been attributed to contaminated disinfectants, intravenous solutions, mechanical ventilators, nebulizers, arterial pressure monitors, urine-measuring containers, and other medical equipment. In this study, although there was no evidence about the mode of transmission of the outbreak, it can be suspected that the bacteria were transmitted via transient carriage on the hands of hospital personnel, which have been previously reported [26] or a bacterial contamination of stethoscopes handled by a health care professional.

\section{CONCLUSION}

It was concluded that ERIC PCR fingerprinting base technique; a simple, rapid and cheap method for the determination of genetic relatedness between Serratia marcescens isolates can be applied for the thorough evaluation of nosocomial outbreaks to detect the source of infection and control the spreading of the outbreak.

\section{ACKNOWLEDGEMENTS}

This study was self-sponsored. The researcher would like to thank Alexander Elliott for the financial support making this project a reality and God for His sustaining grace throughout the successful completion of this research.

\section{AUTHOR CONTRIBUTIONS}

CE made contributions to the study design, data acquisition, analysis and interpretation of data. She also drafted the manuscript. AJV took part in analysis of results and revision of the manuscript. All authors read and approved the final manuscript. All authors declare that they have no competing interests

[3] B.E. Ostrowsky, C. Whitener, H.K. Bredenberg, L.A. Carson, S. Holt, L. Hutwagner, M.J. Arduino, W.R. Jarvis, Serratia marcescens bacteremia traced to an infused narcotic, N. Engl. J. Med. 346 (2002) 15291537.

[4] C. Montagnani, P. Cocchi, L. Lega, S. Campana, K.P. Biermann, C. Braggion, P. Pecile, E. Chiappini, M. De 
Martino, L. Galli, Serratia marcescens outbreak in a neonatal intensive care unit: crucial role of implementing hand hygiene among external consultants, BMC Infect. Dis. 15 (2015) 11.

[5] A. babaei, A. Taheri, Direct Electrochemistry and Electrocatalysis of Myoglobin Immobilized on a Novel Chitosan-Nickel Hydroxide Nanoparticles-Carbon Nanotubes Biocomposite Modified Glassy Carbon Electrode, Anal Bioanal Electrochem, 4(2012) 342 56.

[6] A. Voelz, A. Müller, J. Gillen, C. Le, T. Dresbach, S. Engelhart, M. Exner, C.J. Bates, A. Simon, Outbreaks of Serratia marcescens in neonatal and pediatric intensive care units: clinical aspects, risk factors and management, Int. J. Hyg. Environ. Health. 213 (2010) 79-87.

[7] V. Rabier, S. Bataillon, A. Jolivet-Gougeon, J. Chapplain, A. Beuchée, P. Bétrémieux, Hand washing soap as a source of neonatal Serratia marcescens outbreak, Acta Paediatr. 97 (2008) 1381-1385.

[8] S. Buffet-Bataillon, V. Rabier, P. Bétrémieux, A. Beuchée, M. Bauer, P. Pladys, E. Le Gall, M. Cormier, A. Jolivet-Gougeon, Outbreak of Serratia marcescens in a neonatal intensive care unit: contaminated unmedicated liquid soap and risk factors, J. Hosp. Infect. 72 (2009) 17-22.

[9] P. Villari, M. Crispino, A. Salvadori, A. Scarcella, Molecular epidemiology of an outbreak of Serratia marcescens in a neonatal intensive care unit, Infect. Control Hosp. Epidemiol. 22 (2001) 630-634.

[10] S. Gaisser, V. Braun, The tonB gene of Serratia marcescens: sequence, activity and partial complementation of Escherichia coli tonB mutants, Mol. Microbiol. 5 (1991) 2777-2787.

[11] S.B. Debast, W.J.G. Melchers, A. Voss, J.A.A. HoogkampKorstanje, J. Meis, Epidemiological survey of an outbreak of multiresistantSerratia marcescens by PCR-fingerprinting, Infection. 23 (1995) 267-271.

[12] J. Welsh, M. McClelland, Fingerprinting genomes using PCR with arbitrary primers, Nucleic Acids Res. 18 (1990) 7213-7218.

[13] P.Y. Liu, Y.-J. Lau, B. Hu, J.-M. Shir, M.-H. Cheung, Z.-Y. Shi, W.-S. Tsai, Use of PCR to study epidemiology of Serratia marcescens isolates in nosocomial infection., J. Clin. Microbiol. 32 (1994) 1935-1938.

[14] T.G. Patton, S. Katz, R.J. Sobieski, S.S. Crupper, Genotyping of clinical Serratia marcescens isolates: a comparison of PCR-based methods, FEMS Microbiol. Lett. 194 (2001) 19-25.

[15] R.G.C. Carvalho, I.C. do R.S. Carneiro, M.S. Pinheiro, S. da C. Pinheiro, P.S.R. Azevedo, S.D. dos Santos, A.R.F. da Costa, F.L. de P. Ramos, K.V.B. Lima, Caracterização fenotípica e genotípica de Serratia marcescens provenientes de Unidade Neonatal de Referência em Belém, Pará, Brasil, Rev. Pan-Amazônica Saúde. 1 (2010) 101-106.

[16] P.A. Parment, The role of Serratia marcescens in soft contact lens associated ocular infections: a review, Acta Ophthalmol. Scand. 75 (1997) 67-71.

[17] R. Hertle, Serratia type pore forming toxins, Curr. Protein Pept. Sci. 1 (2000) 75-89.

[18] R. Hertle, The family of Serratia type pore forming toxins, Curr. Protein Pept. Sci. 6 (2005) 313-325.

[19] U. Arslan, I. Erayman, S. Kirdar, S. Yuksekkaya, 0. Cimen, I. Tuncer, B. Bozdogan, Serratia marcescens sepsis outbreak in a neonatal intensive care unit, Pediatr. Int. 52 (2010) 208-212.

[20] Z.-Y. Shi, P.Y. Liu, Y.-J. Lau, Y.-H. Lin, B.-S. Hu, Use of pulsed-field gel electrophoresis to investigate an outbreak of Serratia marcescens., J. Clin. Microbiol. 35 (1997) 325-327.

[21] S. Ktari, F. Mahjoubi, B. Mnif, H. Kallel, M. Bouaziz, A. Hammami, Investigation of three nosocomial outbreaks of Serratia marcescens in an intensive care unit in Sfax-Tunisia, Tunis. Med. 88 (2010) 501-506.

[22] m. Afrasiabi, Z. Rezayati-zad, s. Kianipour, A. babaei, A. Taheri, A Sensor for Determination of Tramadol in Pharmaceutical Preparations and Biological Fluids Based on Multi-Walled Carbon Nanotubes-Modified Glassy Carbon Electrode, JChemSocpak, 35(2013) 1106-12.

[23] C. Sartor, V. Jacomo, C. Duvivier, H. Tissot-Dupont, R. Sambuc, M. Drancourt, Nosocomial Serratia marcescens infections associated with extrinsic contamination of a liquid nonmedicated soap, Infect. Control Hosp. Epidemiol. 21 (2000) 196-199.

[24] G. Bayramoglu, K. Buruk, U. Dinc, M. Mutlu, G. Yilmaz, Y. Aslan, Investigation of an outbreak of Serratia marcescens in a neonatal intensive care unit, J. Microbiol. Immunol. Infect. 44 (2011) 111-115.

[25] V. Milisavljevic, F. Wu, E. Larson, D. Rubenstein, B. Ross, L.M. Drusin, P. Della-Latta, L. Saiman, Molecular epidemiology of Serratia marcescens outbreaks in two neonatal intensive care units, Infect. Control Hosp. Epidemiol. 25 (2004) 719-722.

[26] V. Crivaro, M. Bagattini, M.F. Salza, F. Raimondi, F. Rossano, M. Triassi, R. Zarrilli, Risk factors for extended-spectrum $\beta$-lactamase-producing Serratia marcescens and Klebsiella pneumoniae acquisition in a neonatal intensive care unit, J. Hosp. Infect. 67 (2007) 135-141. 
HOW TO CITE THIS ARTICLE

C. Elliott, A.l Justiz Vaillant, Antimicrobials and Enterobacterial Repetitive Intergenic Consensus (ERIC) Polymerase Chain Reaction (PCR) patterns of nosocomial Serratia marcescens isolates: A One Year Prospective Study (June 2013-May 2014) in a Rural Hospital in the Republic of Trinidad and Tobago, Prog. Chem. Biochem. Res. 2020, 3(2), 114-120.

DOI: $10.33945 /$ SAMI/PCBR.2020.2.4

URL: http://www.pcbiochemres.com/article_106600.html

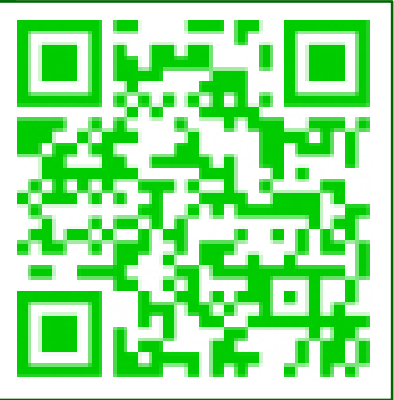

